\title{
Ecoulement multiphasique tridimensionnel par un schéma « faible Mach »-Application au déferlement
}

\author{
Frédéric GOLAY ${ }^{1}$, Philippe HELLUY ${ }^{2}$ \\ ${ }^{1}$ IMATH/MNC, Université du Sud Toulon-Var, ISITV, av. G. Pompidou, BP56, \\ 83162 La Valette du Var,frederic.golay@univ-tln.fr \\ ${ }^{2}$ IRMA - UMR 7501 CNRS/ULP, 7 rue René Descartes, 67084 Strasbourg \\ Cedex,helluy@math.u-strasbg.fr
}

\begin{abstract}
Résumé :
Nous proposons d'approcher numériquement la propagation de vague par un modèle artificiellement compressible à « faible Mach ». La capture de l'interface air-eau est assurée par des schémas volumes finis (Godunov) d'ordre 2 en temps et en espace. L'objectif étant de pouvoir modéliser des déferlements tridimensionnels, l'implémentation est faite dans un code parallèle par sousdomaines et l'équation sur l'énergie est avantageusement abandonnée au profit d'un modèle isotherme conservatif ou non. L'approche, déjà validée par une confrontation numérique/expérimentale est ainsi optimisée en temps de calcul. Nous présenterons deux applications : la propagation de houle et le déferlement tridimensionnel d'une vague sur une pente à $15 \%$.
\end{abstract}

\begin{abstract}
:
We propose to simulate wave propagation by an artificial low Mach compressibility model. The capture of the air-water interface is ensured by a second order finite volume scheme (Godunov) in time and space. As we intend to simulate three-dimensional flow, we use a subdomain method combined with parallel computing. The energy equation is successfully replaced by an isothermal model (conservative or not). This approach, already validated by a numerical/experimental confrontation, is thus optimized in computational time. We will finally describe two applications: wave propagation and solitary wave breaking over an $15 \%$ slope.
\end{abstract}

Mots clés :

Volumes finis - Ecoulement bi-fluide - Soliton - Schéma de Godunov Déferlement - Calcul parallèle 


\section{Introduction}

La simulation numérique de la propagation et du déferlement de vagues solitaires (tsunami) est un domaine de recherche en pleine expansion, voir par exemple l'analyse de GRILLI dans (HELLUY et al., 2005). On approche généralement cet écoulement bi-fluide air-eau, soit par des méthodes intégrales (BIEM) qui prédisent très bien la propagation, le déferlement, mais la reconnexion de l'interface (GRILLI et al., 1989), soit par la résolution des équations de NavierStokes incompressible qui sont très coûteuses en temps de calcul, (VINCENT et al., 2004), (GUIGNARD et al., 2001), (LUBIN et al., 2004) ou (BIAUSSER et al., 2004). Nous proposons d'approcher l'écoulement air/eau par un modèle compressible, bien que l'eau ait un comportement clairement incompressible. L'avantage de cette approche est que l'on peut s'affranchir de l'utilisation de schéma implicite. Une approche, de pré-conditionnement par une compressibilité artificielle (à faible nombre de Mach), a déjà été utilisée avec succès comme par exemple dans (CHANTEPERDRIX et al., 2002) ou dans le cas du déferlement de vague dans (HELLUY et al., 2005) avec comparaison avec d'autres modèles et une expérience de (TANAKA, 1986). Dans le but de réaliser des simulations tridimensionnelles réalistes, nous proposons une amélioration de nos développements par une parallélisation du code, ainsi que l'utilisation d'un modèle isotherme et de schémas d'ordre deux économiques en temps de calcul.

Dans un premier temps, nous exposerons le modèle physique utilisé, l'implémentation numérique, puis nous présenterons les résultats obtenus sur la simulation de propagation de houle bidimensionnelle et sur la propagation et le déferlement d'une vague solitaire tridimensionnelle.

\section{$\underline{\text { 2. Modèles Bi-fluide }}$}

\subsection{Généralités}

Pour simuler l'écoulement air/eau on utilise un modèle d'Euler où les inconnues sont la masse volumique $\rho$, la vitesse $\overrightarrow{\mathrm{u}}$, la pression $\mathrm{p}$ et la fraction d'air $\varphi$. La fraction d'air $\varphi \in[0,1]$ est telle que $\varphi=0$ dans l'eau et $\varphi=1$ dans l'air.

Les équations du modèle à satisfaire sont la conservation de la masse

$\frac{\partial \rho}{\partial t}+\operatorname{div}(\rho \vec{u})=0$,

la conservation de la quantité de mouvement

$\frac{\partial \rho \overrightarrow{\mathrm{u}}}{\partial \mathrm{t}}+\operatorname{div}(\rho \overrightarrow{\mathrm{u}} \otimes \overrightarrow{\mathrm{u}}+\mathrm{p})=\rho \overrightarrow{\mathrm{g}}$,

la conservation de l'énergie 
$\frac{\partial \rho \mathrm{E}}{\partial \mathrm{t}}+\operatorname{div}((\rho \mathrm{E}+\mathrm{p}) \overrightarrow{\mathrm{u}})=\rho \overrightarrow{\mathrm{g}} \cdot \overrightarrow{\mathrm{u}}$

et la convection de la fraction d'air

$\frac{\partial \varphi}{\partial \mathrm{t}}+\overrightarrow{\mathrm{u}} \cdot \nabla \varphi=0$.

L'énergie totale $\mathrm{E}$ est la somme de l'énergie interne spécifique $\varepsilon$ et de l'énergie cinétique spécifique $\|\overrightarrow{\mathrm{u}}\|^{2} / 2$. La pression $p$, suivant le modèle utilisé, est une fonction de la masse volumique, de l'énergie interne et de la fraction d'air : $\mathrm{p}=\mathrm{p}(\rho, \varepsilon, \varphi)$.

\subsection{Lois de pression}

Le nombre de Mach, $\mathrm{M}=\|\overrightarrow{\mathrm{u}}\| / \mathrm{c}$, est le rapport de la vitesse de l'écoulement sur la vitesse du son c. Si le nombre de Mach est uniformément petit, l'écoulement est presque incompressible. Classiquement, on considère que l'écoulement est incompressible si $\mathrm{M}<0.3$. Il n'y a pas de très grande différence entre un écoulement à Mach $\mathrm{M} \approx 1 / 10$ ou à Mach $\mathrm{M} \approx 1 / 1000$ tant que l'on ne s'intéresse pas aux phénomènes acoustiques. La vitesse du son dans l'air est de $400 \mathrm{~m} / \mathrm{s}$ et la vitesse du son dans l'eau est de $1600 \mathrm{~m} / \mathrm{s}$. Comme l'écoulement que l'on veut reproduire est de l'ordre de $1 \mathrm{~m} / \mathrm{s}$ et que l'on s'intéresse principalement à l'écoulement de l'eau, nous imposons une vitesse du son artificielle dans les deux fluides. Cela a pour conséquences bénéfiques, de satisfaire plus facilement la condition de stabilité CFL et de limiter la viscosité numérique et le mauvais comportement des schémas de volumes finis à faible Mach, comme décrit dans (GUILLARD \& VIOZAT, 1999) ou (TURKEL, 1993).

* Pour le modèle énergétique, on résout $(1,2,3,4)$, en utilisant la loi de pression des gaz raides de la forme :

$\mathrm{p}=(\gamma(\varphi)-1) \rho \varepsilon-\gamma(\varphi) \pi(\varphi)$.

Les paramètres de la loi de pression, $\gamma$ et $\pi$, ont l'expression proposée par (SAUREL, 1999),

$$
\begin{aligned}
& \frac{1}{\gamma(\varphi)-1}=\varphi \frac{1}{\gamma_{\mathrm{A}}-1}+(1-\varphi) \frac{1}{\gamma_{\mathrm{w}}-1} \\
& \frac{\gamma(\varphi) \pi(\varphi)}{\gamma(\varphi)-1}=\varphi \frac{\gamma_{\mathrm{A}} \pi_{\mathrm{A}}}{\gamma_{\mathrm{A}}-1}+(1-\varphi) \frac{\gamma_{\mathrm{w}} \pi_{\mathrm{w}}}{\gamma_{\mathrm{w}}-1}
\end{aligned}
$$

où les paramètres $\gamma_{\mathrm{A}}, \pi_{\mathrm{A}}, \gamma_{\mathrm{w}}, \pi_{\mathrm{w}}$, sont déterminés afin que la vitesse du son soit approximativement de $20 \mathrm{~m} / \mathrm{s}$. La vitesse du son est définie par $\mathrm{c}^{2}=\frac{\partial \mathrm{p}}{\partial \rho}+\frac{\mathrm{p}}{\rho^{2}} \frac{\partial \mathrm{p}}{\partial \varepsilon}$. 
soit

$\mathrm{c}=\sqrt{\frac{\gamma(\varphi)(\mathrm{p}+\pi(\varphi))}{\rho}}$

* Pour le modèle isotherme non conservatif, on résout $(1,2,4)$, en utilisant une loi de pression de la forme:

$\mathrm{p}=\mathrm{c}^{2}\left(\rho-\left(\varphi \rho_{\mathrm{A}}+(1-\varphi) \rho_{\mathrm{w}}\right)\right)+\mathrm{p}_{0}$.

où $\mathrm{c}, \rho_{\mathrm{A}}, \rho_{\mathrm{w}}, \mathrm{p}_{0}$ sont les paramètres du modèle.

* Pour le modèle isotherme conservatif (CHANTEPERDRIX, 2004), on résout (1, 2), en utilisant une loi de pression de la forme:

$\mathrm{p}=\varphi \mathrm{p}_{\mathrm{A}}\left(\frac{\varphi \rho}{\alpha}\right)+(1-\varphi) \mathrm{p}_{\mathrm{w}}\left(\frac{(1-\varphi) \rho}{1-\alpha}\right)$

avec

$\mathrm{p}_{\mathrm{A}}=\mathrm{c}_{\mathrm{A}}^{2}\left(\rho-\rho_{0 \mathrm{~A}}\right)+\mathrm{p}_{0}$

$\mathrm{p}_{\mathrm{W}}=\mathrm{c}_{\mathrm{W}}^{2}\left(\rho-\rho_{0 \mathrm{~W}}\right)+\mathrm{p}_{0}$

$\alpha$ tel que $\mathrm{p}_{\mathrm{A}}\left(\frac{\rho_{\mathrm{A}}}{\alpha}\right)=\mathrm{p}_{\mathrm{W}}\left(\frac{\rho_{\mathrm{W}}}{1-\alpha}\right)$

et on remplace l'équation de convection (4) par son équivalent conservatif:

$\frac{\partial \rho \varphi}{\partial \mathrm{t}}+\operatorname{div}(\rho \varphi \overrightarrow{\mathrm{u}})=0$

\section{Modélisation numérique}

\subsection{Schéma volumes finis}

Le problème $(1,2,3,11)$ est un système hyperbolique de loi de conservation, que l'on peut mettre sous la forme

$$
\left\{\begin{array}{c}
\frac{\partial \mathrm{W}(\overrightarrow{\mathrm{x}}, \mathrm{t})}{\partial \mathrm{t}}+\operatorname{div}(\mathrm{F}(\mathrm{W}))=\mathrm{S}(\mathrm{W}) \\
\mathrm{W}(\overrightarrow{\mathrm{x}}, 0)=\mathrm{W}_{0}(\overrightarrow{\mathrm{x}})
\end{array}\right.
$$

où dans le cas d'une loi de pression gaz-raide, par exemple, $W=\langle\rho, \rho \vec{u}, \rho E, \rho \varphi\rangle$. Ce système est approché classiquement par une méthode volumes finis. On décompose le domaine d'étude en cellules où l'on approche le vecteur inconnu $\mathrm{W}$ par une constante représentant la valeur moyenne sur la cellule de volume $\mathrm{C}_{\mathrm{i}}$. En utilisant le théorème de la divergence, on décompose l'intégrale de la divergence en une somme d'intégrales sur toutes les faces de la cellule. Et enfin on discrétise par un schéma temporel explicite, de telle sorte que sur chaque cellule $\mathrm{C}_{\mathrm{i}}$ :

$$
\mathrm{W}_{\mathrm{i}}\left(\mathrm{t}_{\mathrm{n}+1}\right)=\mathrm{W}_{\mathrm{i}}\left(\mathrm{t}_{\mathrm{n}}\right)-\frac{\Delta \mathrm{t}}{\mathrm{C}_{\mathrm{i} \text { faces de }} \mathrm{C}_{\mathrm{i}}} \mathrm{s}_{\mathrm{ij}} \mathrm{F}\left(\mathrm{W}_{\mathrm{i}}\left(\mathrm{t}_{\mathrm{n}}\right), \mathrm{W}_{\mathrm{j}}\left(\mathrm{t}_{\mathrm{n}}\right), \overrightarrow{\mathrm{n}}\right)
$$


où $s_{i j}$ est la surface de la face entre la cellule $i$ et toutes les cellules voisines $j, \vec{n}$ la normale à cette face et $\mathrm{F}$ le flux numérique. Pour plus de détails on peut se reporter par exemple à (ROUY, 2000).

Nous utilisons une méthode volumes finis de type Godunov, le flux numérique est donc approché par la résolution d'un problème de Riemann (différent pour le modèle énergétique ou le modèle isotherme) à chaque interface.

On notera que l'équation (4) est non-conservative, comme dans (ABGRALL, 1996) pour éviter les oscillations de pression. Pour améliorer la précision, nous avons testé les méthodes d'intégration en temps de Runge-Kutta d'ordre 2 (ou Euler point-milieu) et la méthode de MUSCL Hancock (TORO, 1999). Comme limiteur de pente, nous avons testé la méthode de Barth car elle très simple, et la méthode WLSQR (weighted least square) (FURST \& KOZEL, 2002). Pour plus de détails, le lecteur pourra se référer à (GOLAY \& HELLUY, 2007).

\subsection{Parallélisation et temps de calcul}

Afin de réaliser des simulations tridimensionnelles réalistes, un grand nombre de degrés de liberté est nécessaire, c'est pourquoi nous avons optimisé nos développements par une approche parallèle. Ces travaux ont été intégrés dans un code de calcul développé conjointement à l'université de Toulon, l'université de Strasbourg et la société Principia R\&D. Le code a été conçu sur une architecture logicielle qui autorise les maillages non structurés et une résolution sur machine parallèle. Nous avons donc utilisé une approche orienté objet (en Fortran 90). Grace à une formulation compressible, nous profitons de l'écriture par un schéma explicite, qui nous évite d'avoir à résoudre des systèmes linéaires, si bien que la décomposition en sous domaines en est facilitée.

Pour chaque cellule on définit un objet 'état' qui porte toutes les inconnues sur la cellule. L'élément le plus important de l'algorithme est alors l'objet 'face' qui est constitué essentiellement de pointeurs vers les 'états' des deux cellules voisines et de la normale. Ainsi, toute étape de l'algorithme de résolution du problème volumes finis, repose essentiellement sur des boucles sur toutes les faces du maillage, connaissant les 'états' au début du pas de temps. En conséquence la décomposition en sous-domaines ne nécessite, au début de chaque pas de temps, que la connaissance des 'états' des cellules voisines au domaine. On a, en quelque sorte, un recouvrement d'une cellule entre les domaines. Après validation de cette approche sur des cas tests de type «tube à chocs», nous avons simulé la propagation d'un soliton sur fond plat sur 1,2,4 ou 8 processeurs Itanium II, afin de vérifier que la performance de la décomposition par sous domaine, n'était pas altérée par les temps de communication entre les process. Dans la figure ci-après 
(Figure 1), on observe le rendement (Temps sur 1 processeur /Temps cumulé de $n$ processeur) pour le temps cpu (sans les communications et le système) et le temps de calcul global (avec communication et éventuellement d'autres jobs). On observe de façon tout à fait surprenante que le rendement est supérieur à 1 ! Cela est dû au fait, d'une part que les communications entre process sont très faibles et d'autre part que sur chaque sous-domaine, le calcul étant plus petit, on a une meilleure gestion de la mémoire cache.

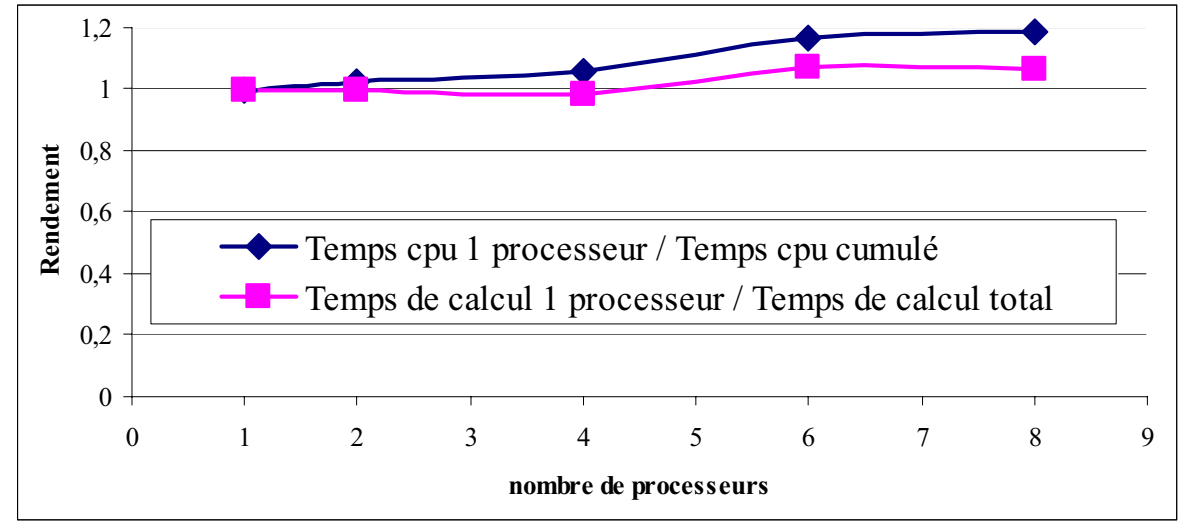

Figure 1. Rendement du parallélisme

A cette occasion, nous avons également comparé les résultats obtenus avec les modèles énergétiques, isotherme non conservatif et isotherme conservatif. Les résultats sont absolument identiques car l'équation sur l'énergie n'influence pas la physique du problème. En temps de calcul par contre on constate une grande disparité de temps CPU: modèle gaz-raide $3 \mathrm{~h} 03 \mathrm{~min}$, modèle isotherme non conservatif $1 \mathrm{~h} 25 \mathrm{~min}$ et modèle isotherme conservatif $1 \mathrm{~h} 18 \mathrm{~min}$. Il est compréhensible d'être plus économique avec le modèle isotherme car on résout une équation de moins. Le facteur 2 que l'on observe avec le modèle énergétique s 'explique par le fait que des oscillations inutiles de la pression, pénalisent grandement la condition de CFL dans le cas du modèle gaz-raide et génèrent donc un trop petit pas de temps. Le faible écart entre le modèle conservatif et non conservatif s'explique par le fait que l'on doit calculer deux fois le flux numérique pour l'équation de transport qui est non conservative.

\section{Applications}

\subsection{Propagation de houle}

La propagation d'une vague solitaire ayant déjà été validée par une confrontation expérimentale (HELLUY et al., 2005), nous proposons de tester la propagation d'une houle de Stokes, pour vérifier par la suite l'influence du vent (programme LEFE-IDAO). Dans un premier temps, nous cherchons à vérifier que la simulation est suffisamment précise pour conserver la propagation de la houle. 
On introduit des conditions aux limites périodiques aux bords du domaine et on initialise par une houle de Stokes (hauteur 1, amplitude 0.05, nombre d'onde $2 \pi$ ). Le domaine d'étude est découpé en quatre sous domaines sur environ 80000 cellules. On propage la houle pendant 2 s (on traverse un peu plus de 2 domaines) pour un temps de calcul de 38 minutes sur quatre processeurs. Les résultats sont encourageants en temps de calcul, mais peuvent être améliorés en termes de qualité d'interface comme on le constate sur la figure 2 où est représentée la houle à l'instant initial et après $2 \mathrm{~s}$. Afin de diminuer la diffusion à l'interface nous allons développer une contraction de l'interface sur le principe de (OLSSON \& KREISS, 2005), avant de tester l'influence du vent.

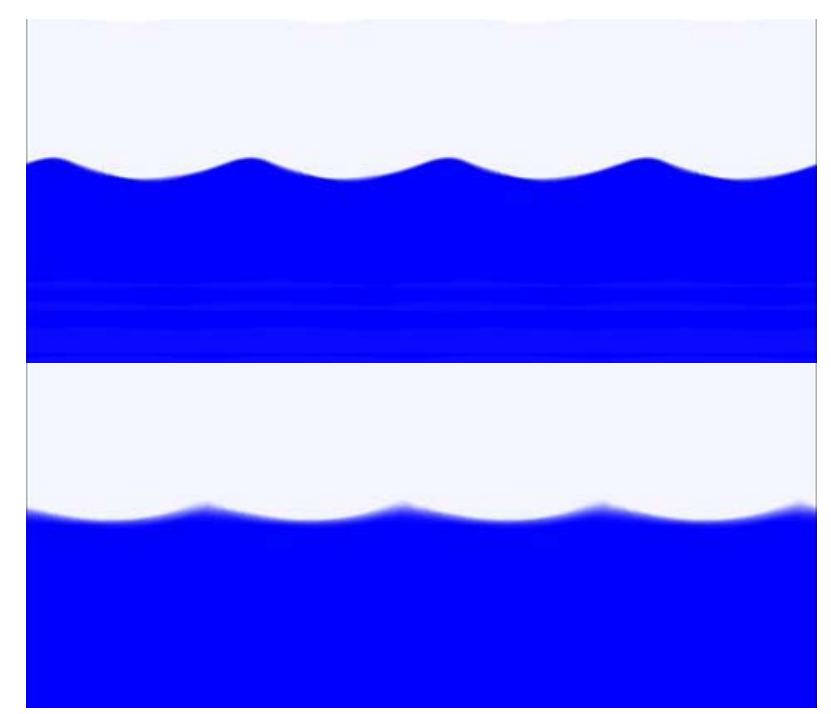

Figure 2. Propagation de houle : Fraction d'eau bleu/eau blanc/air à l'instant initial et après 2 secondes

\subsection{Propagation et déferlement d'un soliton}

Afin de vérifier la validité de notre approche, nous simulons la propagation puis le déferlement d'une vague solitaire telle que décrit dans (GRILLI et al., 2001). La vague est initialisée par une solution de (TANAKA, 1986) comme dans (HELLUY et al., 2005), se propage sur fond plat jusqu'à $\mathrm{x}=5,225 \mathrm{~m}$, puis se cambre sur une pente à $15 \%$ avant de déferler. En imposant une condition de symétrie sur le milieu de la bosse, on ne maille qu'une partie de la vague. Les résultats obtenus sont tout à fait comparables à ceux de (BIAUSSER et al., 2004), mais dans un temps de calcul bien moindre. : $96 \mathrm{~h}$ de calcul sur 8 processeurs pour environ 2 millions de cellules. Le modèle utilisé était isotherme non conservatif, d'ordre 2 en espace et en temps. Sur les figures ci-après est tracée la surface de 
l'isovaleur $\varphi=0.5$, ce qui représente l'interface air/eau, sauf lors du déferlement ou il y a en partie mélange des 2 phases.

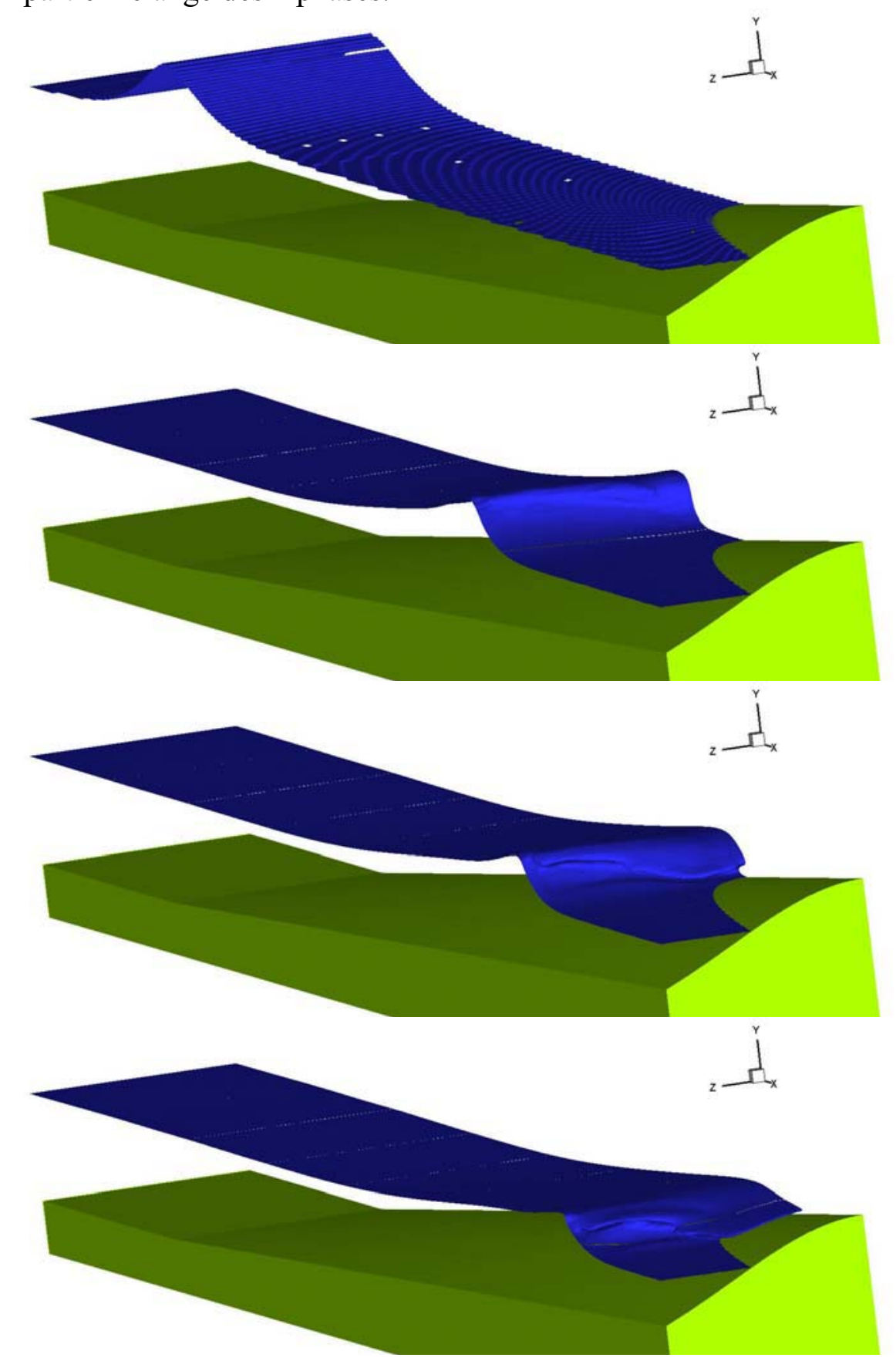

Figure 3. $\varphi=0.5$ Propagation et déferlement tridimensionnel d'une vague solitaire, à $t=0 \mathrm{~s}, t=3.5 \mathrm{~s}, t=3.5 \mathrm{~s}$ et $t=4 \mathrm{~s}$ 


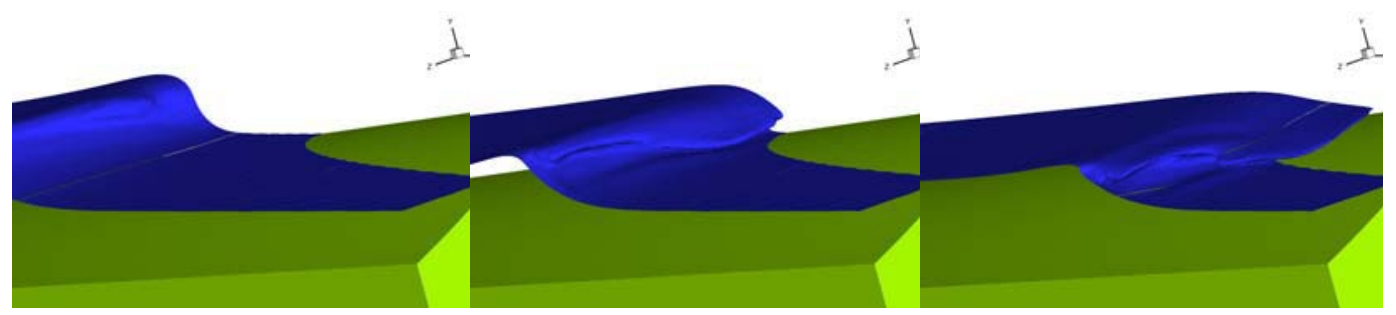

Figure 4. $\varphi=0.5$ Déferlement tridimensionnel d'une vague solitaire, $t=3.5 \mathrm{~s}$, $t=3.5 \mathrm{~s}$ et $t=4 \mathrm{~s}$

\section{Conclusion}

Nous avons proposé une méthode volumes finis pour approcher les écoulements bi-fluide, en introduisant une compressibilité artificielle. Cette compressibilité est introduite pour obtenir des conditions de CFL moins contraignantes et une plus grande précision. Le développement d'une loi isotherme et l'approche par sousdomaines a permis de diminuer grandement les temps de calcul. En conséquence il devient possible de réaliser des simulations tridimensionnelles réalistes, comme nous l'avons vérifié sur le déferlement d'une vague solitaire. Cette approche est donc une alternative à la résolution des écoulements incompressibles coûteux en temps de calcul. Cependant, il est nécessaire d'améliorer encore la précision de l'interface, comme nous l'avons constaté sur la propagation de la houle. Nos développements futurs s'orientent donc vers la montée en ordre et des techniques de compression d'interface.

1 ABGRALL, R. (1996) How to prevent pressure oscillations in multicomponent flow calculations: a quasi-conservative approach. Journal of Computational Physics, 125(1):150-160.

2 BIAUSSER, B., S. GUIGNARD, S., MARCER, R. \& FRAUNIE, P (2004). 3D two phase flows numerical simulations by SL-VOF method. International Journal for Numerical Methods in Fluids, 45(6):581-604.

3 CHANTEPERDRIX, G., VILLEDIEU, P. \& VILA, J.-P. (2002) A compressible model for separated two-phase flows computations. In ASME Fluids Engineering Division Summer Meeting. ASME, Montreal, Canada.

4 CHANTEPERDRIX, G. (2004), Modélisation et simulation numérique d'écoulements diphasiques à interface libre. Application à l'étude des mouvements de liquides dans les réservoirs de véhicules spatiaux. Thèse de doctorat, Ecole Nationale Supérieure de l'Aéronautique et de l'Espace.

5 FÜRST, J., KOZEL, K. (2002). Second and third order weighted ENO scheme on unstructured meshes. In Finite volumes for complex applications, III (Porquerolles), pages 723-730 (electronic). Lab. Anal. Topol. Probab. CNRS, Marseille. 
6 GRILli S.T., Skourup J., and I. A. Svendsen I. A. (1999), An efficient boundary element method for non-linear water waves. Engng. Analysis with Boundary Elements, 6(2):97-107.

7 GUIGNARD, S., R. MARCER, R., REY, V., KHARIF, C. \& FRAUNIÉ, P. (2001). Solitary wave breaking on sloping beaches: 2-D two phase flow numerical simulation by SL-VOF method. European Journal of Mechanics. B. Fluids, 20(1):57-74.

8 GUILLARD, H. \& VIOZAT, C. (1999). On the behaviour of upwind schemes in the low Mach number limit. Computers \& Fluids. An International Journal, 28(1):63-86.

9 GOLAY, F., HELlUY, P., (2007). Numerical schemes for low Mach wave breaking. Int. J. of computational Fluid Dynamics, vol.21 n², pp 69-86.

10 GRILLI, S. T., GUYENNE, P., AND DIAS, F. (2001), A fully nonlinear model for three-dimensional overturning waves over arbitrary bottom, Intl J.Numeric. Meth. Fluids, 35 (1), 829-867.

11 HELlUY, P., GOLAY, F. \& al (2005). Numerical simulation of wave breaking. M2AN. Mathematical Modelling and Numerical Analysis, 39(3):591607.

12 LUBIN P., VINCENT S., GRILLI S.T., GILBERT R.W., DREVARD D., REY V., FRAUNIE P., MARCER R., ASTRUC D., LEGENDRE D., DUVAL M., KIMMOUN O., BRANGER H;, GUIGNARD S., ABADIE S., Simulations numériques et expérimentales d'un soliton se propageant sur une plage inclinée. VIII ${ }^{\mathrm{èmes}}$ J. Nat. Génie Civil - Génie Côtier, Compiègne, 7-9 septembre 2004.

13 OLSSON E., KREISS G. (2005), A conservative level set method for two phase flow, J.of Comp. Phy., 210, pp225-246.

14 ROUY S. (2000), Modélisation mathématique et numérique d'écoulements diphasiques compressibles - application au cas industriel d'un générateur de gaz, thèse de l'Université du Sud Toulon-Var.

15 TANAKA, M. (1986). The stability of solitary waves. Physics of Fluids, 29:650-655.

16 TORO, E. F. (1999), Riemann solvers and numerical methods for fluid dynamics, 2nd edition, Springer.

17 TURKEL, E. (1993). Review of preconditioning methods for fluid dynamics. Applied Numerical Mathematics, 12(1-3):257-284. Special issue to honor Professor Saul Abarbanel on his sixtieth birthday (Neveh).

18 VINCENT, S., CALTAGIRONE,J.-P., LUBIN, P. \& RANDRIANARIVELO, T.N. (2004). An adaptative augmented Lagrangian method for three-dimensional multimaterial flows. Computers and Fluids, 33(10):1273-1289. 